\title{
Unusual Structural Transformation in LiMn2O4 Cathode Revealed by Atomic- Scale STEM Characterization
}

\author{
Lin $\mathrm{Gu}^{1}$
}

${ }^{1 .}$ Beijing National Laboratory for Condensed Matter Physics, Institute of Physics, Chinese Academy of Sciences, Beijing, China.

The rapid development of portable electronics and hybrid electric vehicle (HEV) markets promotes the research of electric energy storage systems. Among them, lithium-ion batteries (LIBs) have attracted intense attention, due to their high energy density and long cycle lifetime. The demand for higher energy density LIBs facilitate the study of various electrode materials. Performance optimization of electrode materials necessitates information about structural evolution of electrode materials at an atomic scale. However, limited understanding for the structural evolution of electrode materials at atomic resolution, especially the $\mathrm{Li}$ ions distribution and arrangement at the atomic level, underlying electrochemical process substantially hinders our exploration of reaction mechanism and further performance optimization.

The annular-bright-field (ABF) imaging in aberration-corrected scanning transmission electron microscopy (STEM) allows simultaneous imaging of light and heavy elements, providing an unprecedented opportunity to probe the nearly equilibrated local structure of electrode materials after electrochemical cycling at atomic resolution. A JEM-ARM200F STEM operated at $200 \mathrm{kV}$ and equipped with double aberration-correctors for both probe-forming and imaging lenses was used to perform $\mathrm{ABF}$ and high angle angular dark-field (HAADF) imaging with the acceptance semiangle 12 25 mrad and 90-370 mrad, respectively.

The most interesting cathode materials for lithium-ion batteries are spinel $\mathrm{LiMn}_{2} \mathrm{O}_{4}$ and its derivatives, e.g., $\mathrm{LiNi}_{0.5} \mathrm{Mn}_{1.5} \mathrm{O}_{4}$, due to their low cost and nontoxicity. However, the cycling performance of these cathode materials degrades due to structural distortion during electrochemical cycling, particularly at elevated temperatures. We investigated the atomic-level surface structure of $\mathrm{LiMn}_{2} \mathrm{O}_{4}$ by heat-treatments in various atmospheres, finding similar surface spinel-to layered structural transformation only for $\mathrm{LiMn}_{2} \mathrm{O}_{4}$ heat-treated in argon atmosphere for a few minutes (Figure 1). It suggested that the spinel-tolayered transformation is made possible via migration of $\mathrm{Mn}$ ions from octahedral sites to lithium tetrahedral sites, forming an intermediate $\mathrm{LiMn}_{3} \mathrm{O}_{4}$ structure, followed by a further shift of some of $\mathrm{Mn}$ ions from the $\mathrm{LiMn}_{3} \mathrm{O}_{4}$ back to the octahedral sites to form the layered-like structure. The distortion of the surface regions can be extended to the whole bulk by heat-treatment for 300-600 min, ultimately enabling us to identify the bulk-level structure as layered $\mathrm{Li}_{2} \mathrm{MnO}_{3}$ (Figure 2). This work demonstrates the critical role of $\mathrm{Mn}^{3+}$ in controlling the kinetics of the structural transformation in spinel $\mathrm{LiMn}_{2} \mathrm{O}_{4}$ and suggests heat-treatment in argon as a convenient method to control the surface oxygen loss and consequently reconstruct the atomic-level surface structure [2]. 
References:

[1] Ben et al, Acs Appl. Mater. Inter. 9 (2017), p. 35463.

[2] The authors acknowledge funding from the National Natural Science Foundation of China. Prof. X. J. Huang and L. B. Ben are thanked for many useful discussions and contributions to this work.

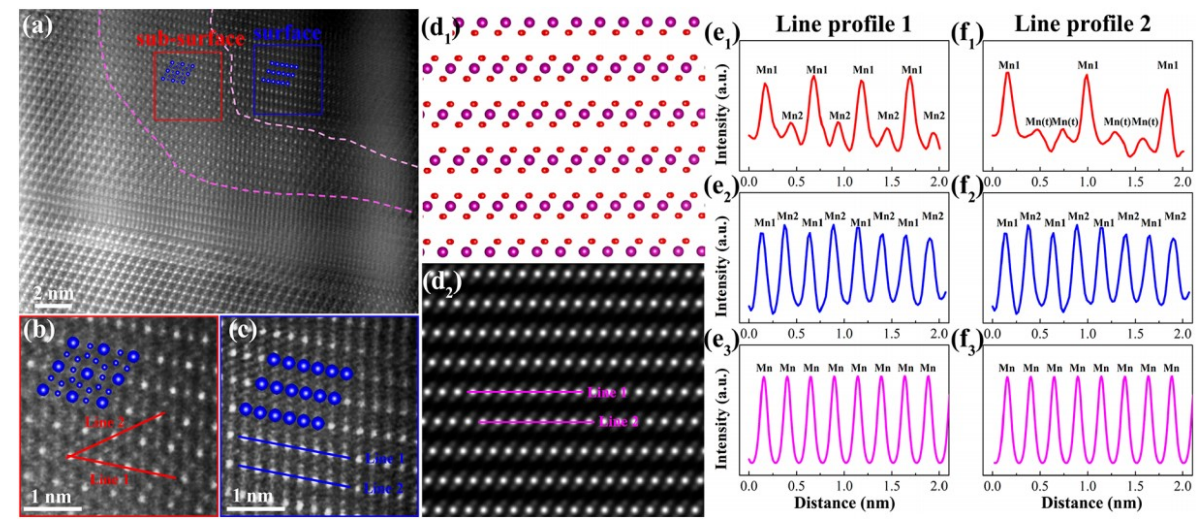

Figure 1. (a) Typical STEM-HAADF image of high-voltage cycled LMO (16th cycle, fully charged). (b, c) Enlarged images of the surface and subsurface regions, corresponding respectively to the red and blue boxes in panel (a). (d1) Crystal structure of the layered (e.g., $\mathrm{Li}_{2} \mathrm{MnO}_{3}, \mathrm{C} 2 / \mathrm{m}$ ) viewed along the [100] direction. Mn atoms (purple) occupy octahedral sites. $\mathrm{O}$ atoms are depicted in red. (d2) Corresponding simulated STEM-HAADF image. (e, f) Line profiles corresponding to the red lines in panel (b), blue lines in panel (c), and black lines in panel (d), respectively. (Schematic lattice structures are overlaid in panels a-c.)

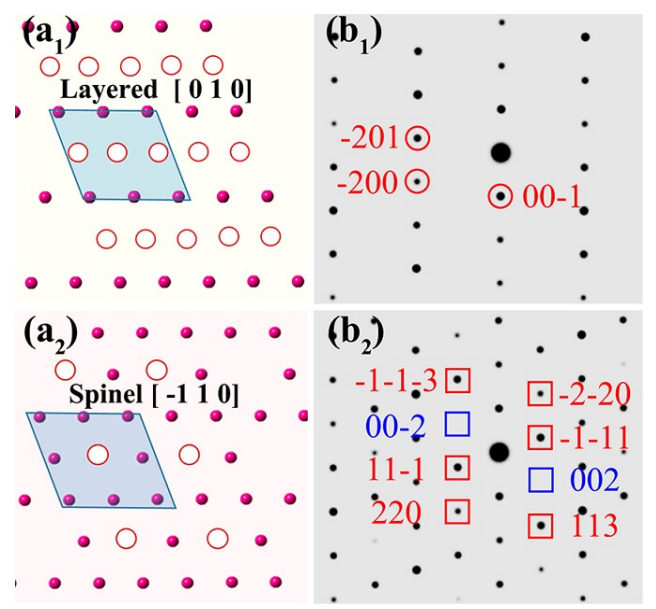

Figure 2. Schematics showing the $\mathrm{Mn}$ sublattice in (a1) layered structure, e.g, $\mathrm{Li}_{2} \mathrm{MnO}_{3}(\mathrm{C} 2 / \mathrm{m})$ viewed along the $\left[\begin{array}{lll}0 & 1 & 0\end{array}\right]$ direction and (a2) spinel LMO (Fd-3m) viewed along the [1-1 0] direction. (b1) Simulated electron diffraction patterns corresponding to the layered structure and (b2) the spinel structure. 